\title{
VII. Untersuchungen in der Reihe des Indazols;
}

ron $K$. Fries and E. Roth.

Das 6-Oxyindazol (I), das wir einem Vergleich mit dem $\beta$-Naphthol unterzogen, stellten wir aus 6 -Amidoindazol durch Diazotieren und Verkochen dar. ${ }^{3}$ )

Die Einwirkung begrenzter Mengen von Chlor und von Brom auf die Oxyverbindung führt zunächst zu Monohalogenderivaten. Dem so entstehenden Chloroxyindazol kommt die Formel II zu, Chlor nimmt also den Platz zwischen der Hydroxylgruppe und dem zweiten Ring ein, wie beim $\beta$-Naphthol. Es folgt das aus dem gleich zu schildernden Verhalten der Chlorverbindung bei der weiteren Chlorierung und bei der Einwirkung von Salpetersäure. Man kann nicht annehmen, daß das Brom einen anderen Platz aufsucht als das Chlor.

I.

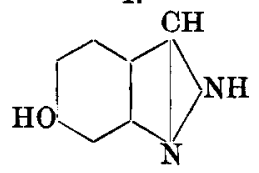

II.

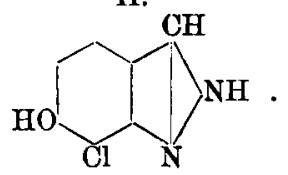

Läßt man äquimolekulare Mengen Chlor und 7 -Chlor6 -oxyindazol aufeinander wirken, dann gehen zwei Reaktionen nebeneinander her. Einmal bildet sich das Indazolketochlorid (III) und weiter das 5, 7 -Dichloroxyindazol (IV).

III.<smiles></smiles>

IV.<smiles>OCC1C2CCCC(N2)C1O</smiles>

Die Entstehung des Ketochlorids (III), das bei der Reduktion in Chloroxyindazol zurückgeht, kann als Beweis dafür angesehen werden, daß das zuerst ein-

1) Nach Witt, Nölting u. Grandmougin, Ber. d. d. chem. Ges. 23, 3636 (1890); 25, 3151 (1892). 
tretende Chloratom die 7-Stellung einnimmt. Denn dah ein 5-Chlor-6-oxyindazol (a) bei der weiteren Linwirkung von Chlor ein Dichlorketochlorid bilden soll, dem dann die Formel b) zukäme, das stände einzig da.

a)<smiles>OC1C2CCCC1NC2</smiles>

b)

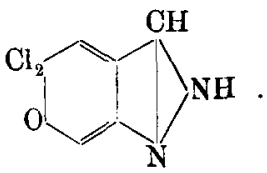

In der Bildung des Ketochlorids zeigt das Oxyindazol Verwandtschaft mit dem $\beta$-Naphthol, die Entstehung des Dichlorsubstitutionsproduktes fübrt es dagegen an die Seite der Phenole.

Dieser Zwittercharakter des Indazols tritt auch bei der Umsetzung des Chloroxyindazols (II) mit Salpetersäure hervor. Wie bei dem 1-Chlor-2-naphthol bildet sich ein Nitrochlorketon, das 7-Nitro-7-chlor-6-keto-6,7-dihydroindazol (V). Daneben aber entsteht - wenn auch nur in geringer Menge - ein Nitrochloroxyindazol (VI); hierbei ist die Reaktion also in der gleichen Weise vor sich gegangen, wie es bei entsprechenden Benzolderivaten der Fall ist.

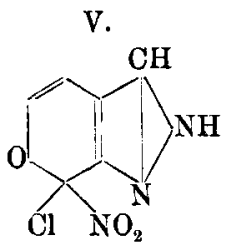

VI.

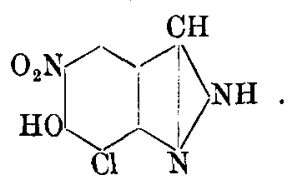

Sehr auffällig ist es nun, daß das T-Brom-6-oxyindazol (VII) mit Salpetersäure keine nachweisbare Menge eines Nitrobromketons liefert. Nan erhält vielmehr ausschließlich ein Nitrobromoxyindazol, dem wir die Formel VIII geben.

VII.

VIII.
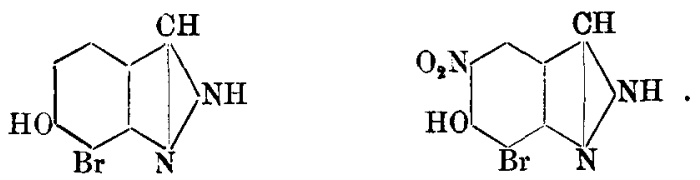
Dieses unterschiedliche Verhalten des Chlor- und des Bromoxyindazols ist nur schwer verständlich, es müßte denn die oben gemachte Voraussetzung, daß Chlor und Brom, bei der Bildung der Monohalogenderivate des Oxyindazols, denselben Platz aufsuchen, falsch sein. Ist diese Annahme auch unwahrscheinlich, so soll sie doch geprüft werden.

Das Nitrochlorketoindazol (V) geht, wie die entsprechende Verbindung der Naphthalinreihe, beim Kochen in indifferenten Lösungsmitteln in ein Orthochinon, das 6,7-Indazolchinon (VIII), über, aus dem durch Reduktion das 6,7-Dioxyindazol (IX) erhältlich ist.

VIII.<smiles>NC1NC2=C1C=COO2</smiles>

IX.

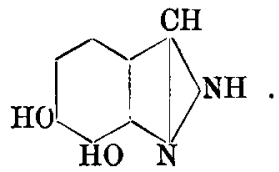

Schon früher wurde darauf hingewiesen ${ }^{\mathbf{l}}$ ), dab das 7-Chloroxyindazol im Gegensatz zu den entsprechenden Abkömmlingen des Naphthalins, Azimidobenzols und Pseudoazimidobenzols, mit Diazoverbindungen zu Azofarbstoffen kuppelt, daß das aber nicht ohne weiteres gegen eine Analogie mit dem Naphthalin zeugt, da Indazol selbst fähig ist mit Diazoverbindungen zu kuppeln. Eine eingehende Untersuchung der Kupplungsprodukte, die für Vergleichszwecke nötig ist, mußte wegen der Schwierigkeiten, die mit der Materialbeschaffung verknüpft sind, verschoben werden.

Aus den wenigen, im vorstehenden geschilderten Versuchsergebnissen ersieht man jedenfalls das eine, schon früher betonte, daß die in bezug auf das chemische Verhalten überraschend große Übereinstimmung mit dem $\beta$-Naphthol, die die entsprechenden Oxyderivate des Azimidobenzols und des Pseudoazimidobenzols auszeichnet,

1) Diese Annalen 389, 313 (1912). Dort hat sich ein sinnentstellender Druckfehler eingeschlichen. In der zweiten Zeile von unten ist an Stelle von "nicht" „noch" zil lesen. 
bei dem Oxyindazol nicht mehr vorhanden ist. Andererseits steht es dem Naphthol aber viel näher, als z. B. das Umbelliferon oder als das isomere Oxybenzimidazol, worüber demnächst berichtet werden soll.

Die Frage soll noch geprüft werden, wieweit die in der Reihe des 6-Oxyindazols gewonnenen Ergebnisse bestätigt werden, wenn das 5-Oxyindazol einem Vergleich mit dem $\beta$-Naphthol unterzogen wird.

\section{Experimenteller Teil.}

$$
\text { 6-Oxyindazol, }
$$

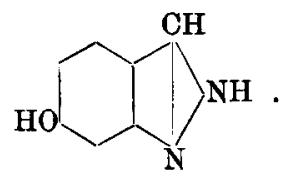

Das 6-Oxyindazol ist bereits von Witt und Nölting ${ }^{1}$ ) aus dem entsprechenden Amidoindazol durch Diazotierung und Verkochen gewonnen worden. Wir geben im folgenden eine genaue Vorschrift, die leidliche Ausbeuten liefert.

Man löst $3 \mathrm{~g} 6$-Amidoindazol in einem Gemisch von $3 \mathrm{ccm}$ konz. Schwefelsäure und $20 \mathrm{ccm}$ Wasser, kühlt auf $0^{\circ}$ ab und fügt dann tropfenweise unter Schütteln eine Lösung von $1,57 \mathrm{~g}$ Natriumnitrit in $5 \mathrm{ccm} W$ asser hinzu. Nach weiterer Zugabe von $3 \mathrm{ccm}$ konz. Schwefelsäure erhitzt man über freier Flamme, bis lebhafte Stickstoffentwicklung beginnt, und setzt dann mit kleiner Flamme das Erhitzen fort, bis die Gasentwicklung nahezı beendet ist, was etwa $1 \frac{1}{2}$ stunde währt. Von einem roten Nebenprodukt, das sich ausscheidet, filtriert man nun $a b$ und neutralisiert, unter sehr guter Kühlung, mit Ammoniak. Die Oxyverbindung, durch braune Beimengungen verunreinigt, scheidet sich ab. Ein kleiner Teil bleibt in der Mutterlauge gelöst: man gewinnt ihn durch Ausäthern. Aus dem Rohprodukt erhält man

1) Ber. d. d. chem. Ges. 23, 3636 (1890); 25, 3152 (1892). 
durch Auskochen mit Wasser das reine Oxyindazol. Die Ausbeute schwankt $z$ wischen $1,5 \mathrm{~g}$ und $1,75 \mathrm{~g}$.

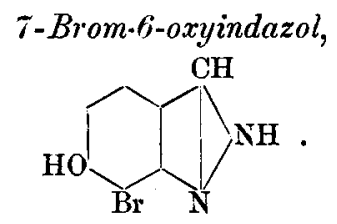

$\mathrm{Zn}$ der mit Eis gekühlten Lösung von 2,2 $\mathrm{g}$ Oxyindazol in $10 \mathrm{ccm}$ Eisessig setzt man nach und nach eine Mischung aus $2,6 \mathrm{~g}$ Brom und $3 \mathrm{ccm}$ Eisessig. Das Bromierungsprodukt scheidet sich größtenteils aus, den Rest gewinnt man durch teilweises Abdunsten der Mntterlauge. Das Reaktionsprodukt ist das bromwasserstoffsaure Salz des Bromoxyindazols. Znr Überführung in die freie Verbindung löst man es in wenig Wasser und fällt die Oxyverbindung durch Natriumacetat. Zur Reinigung wird sie aus Chloroform umkrystallisiert, woraus sie in Nadeln erhalten wird, die bei $182^{\circ}$ schmelzen. Leicht löslich in Alkohol und in Eisessig, schwerer in Benzol, Chloroform und Wasser, schwer in Benzin. Konz. Schwefelsänre nimmt es farblos auf.

$0,1414 \mathrm{~g}$ gaben $0,1245 \mathrm{AgBr}$.

Ber. für. $\mathrm{C}_{7} \mathrm{H}_{5} \mathrm{ON}_{2} \mathrm{Br}$

$\mathrm{Br}$

37,52

Gef.

37,47

$$
\text { -Chlor-6-oxyindazol, }
$$

In eine Lösung von $1,33 \mathrm{~g}$ Oxyindazol in $15 \mathrm{ccm}$ Eisessig leitet man Chlor ein, das aus $0,63 \mathrm{~g}$ Kaliumpermanganat und Salzsäure entwickelt wird. Der größte Teil des Eisessigs wird dann verdampft und durch Zusatz von Wasser und Natriumacetat das Chloroxyindazo] gefällt. In Eisessig und in Alkohol ist es leicht löslich, schwer in Benzol und in Benzin. Zur Umkrystalli- 
sation eignet sich Wasser, aus dem sich die Chlorverbindung in Nadeln ausscheidet, die bei $184^{\circ}$ schmelzen.

$0,1045 \mathrm{~g}$ gaben $0,0892 \mathrm{AgCl}$.

Ber. für $\mathrm{C}_{7} \mathrm{H}_{5} \mathrm{ON}_{2} \mathrm{Cl}$

Gef.

$\mathrm{Cl}$

21,03

21,11

Das in Wasser ziemlich leicht lösliche salzsaure Salz bildet lanzettenförmige Krystalle, die 2 Mol. Krystallwasser enthalten (ber. Cl 29,41; gef. 29,17), die bei $100^{\circ}$ noch nicht abgegeben werden. Die Krystalle schmelzen bei $206^{\circ}$ unter Zersetzung. Krystallisiert man das wasserhaltige Salz aus Eisessig um, dann scheiden sich feine Nadeln aus, die Krystall-Eisessig enthalten (ber. $\mathrm{Cl} 26,75$; gef. 26,67).

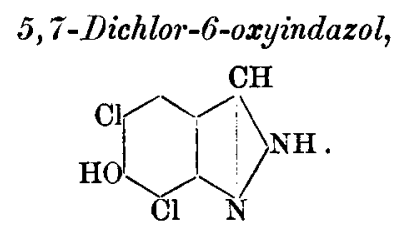

Leitet man in die aus $1,68 \mathrm{~g}$ Chloroxyindazol und $20 \mathrm{ccm}$ Eisessig bereitete Lösung das aus $0,63 \mathrm{~g}$ Kaliumpermanganat and Salzsäure entstehende Chlor ein, dann wird das Chlor restlos aufgenommen. Die Lösung enthält ein Ketochlorid, das sich einmal daran erkennen läßt, daß es aus Jodkalium Jod frei macht, und weiterhin daran, daß die Lösung größere Mengen von Zinnchlorür verbraucht. Das Ketochlorid kann aber nicht das einzige Produkt der Reaktion sein, denn bei der Reduktion dürfte aus ihm nur das Chloroxyindazol zurückgebildet werden:

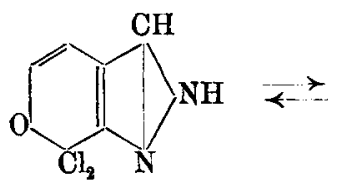<smiles>OC1CCC2C(O)C3NC1C23</smiles>

Entzinnt man aber die mit Zinnchlorür reduzierte Lösung mittelst Schwefelwasserstoff, dampft das Lösungsmittel zum größten Teil ab und fällt dann das Reaktions- 
produkt mit Natriumacetat, so erhält man ein Produkt, das im rohen Zustand rund 27 Proz. Chlor enthält (ber. für $\mathrm{C}_{7} \mathrm{H}_{5} \mathrm{ON}_{2} \mathrm{Cl}$ 21,11 Proz., für $\mathrm{C}_{7} \mathrm{H}_{4} \mathrm{ON}_{2} \mathrm{Cl}_{2}$ 34,93 Proz.) und das sich als ein Gemisch von Mono- und Dichloroxyindazol erweist. Durch mehrmaliges Umkrystallisieren ans Wasser, in dem sich die Dichlorverbindung schwerer löst als das Monochlorderivat, gelingt wenigstens eine teilweise Trennung. Aus dem in Wasser schwerer löslöslichen Teil erhält man durch öfteres Umkrystallisieren aus etwas verdünntem Eisessig die Dichlorverbindung rein. Sie bildet Nadeln, die bei $203^{\circ}$ schmelzen. In Alkohol und in Eisessig löst sie sich leicht, schwer in Benzol, noch schwerer in Wasser und in Benzin. $0,0915 \mathrm{~g}$ gaben $0,1289 \mathrm{AgCl}$.
Ber. für $\mathrm{C}_{7} \mathrm{H}_{4} \mathrm{ON}_{2} \mathrm{Cl}_{2}$ $\mathrm{Cl}$ 34,93
Gef. 34,83

Das Dichloroxyindazol löst sich in Natronlauge ohne Veränderung, im Gegensatz zu dem rohen Chlorierungsprodukt, das sich damit schmutzig braun färbt. Durch Salpetersäure wird es zu einem leuchtend roten o-Chinon oxydiert.

Alle Versuche, durch Zusatz von Wasser oder Salzsäure zum Lösungsmittel oder durch Wechsel des Lösungsmittels und der Temperatur die Chlorierung des Monochloroxyindazols so zu leiten, daß entweder nur das Ketochlorid oder nur das Dichlorsubstitutionsprodukt entsteht, waren ohne Erfolg. Stets bilden sich beide Verbind ungen nebeneinander.

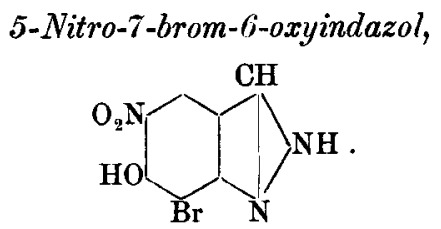

Die Lösung von $0,5 \mathrm{~g}$ Bromoxyindazol in $6 \mathrm{ccm}$ kaltem Eisessig versetzt man mit 10 Tropfen rauchender Salpetersäure $(1,52)$. Zunächst scheidet sich das salpeter- 
saure Salz des Indazolderivats ab, das aber beim Erwärmen im Wasserbad in Lösung geht. Man kühlt dann sofort ab und versetzt tropfenweise mit Wasser, bis sich das Nitrierungsprodukt in gelben Kryställchen abscheidet. Es erleidet weder beim Erhitzen in hochsiedenden Lösungsmitteln, noch beim Lösen in Natronlauge eine Veränderung. Ein Nitroketon, wie es bei der Nitrierung des Chloroxyindazols entsteht, liegt also nicht vor, sondern ein Nitrophenol.

Aus Eisessig krystallisiert die Verbindung in gelben Nadeln, die bei $239^{\circ}$ unter Zersetzung schmelzen. In Alkohol löst sie sich leicht, schwerer in Benzol, schwer in Benzin und in Wasser.

$0,1229 \mathrm{~g}$ gaben $17,6 \mathrm{ccm}$ Stickgas bei $15^{\circ}$ und $744 \mathrm{~mm}$ Druck. $0,1220 \mathrm{~g} " 0,0883 \mathrm{AgBr}$.

$\begin{array}{ccc} & \text { Ber. für } \mathrm{C}_{7} \mathrm{H}_{4} \mathrm{O}_{3} \mathrm{~N}_{9} \mathrm{Br} & \text { Gef. } \\ \mathrm{N} & 16,32 & 16,37 \\ \mathrm{Br} & 30,98 & 30,80\end{array}$

Die Nitroverbindung bildet orangefarbene Alkalisalze, die sich in Wasser ziemlich leicht lösen, aus diesen Lösungen durch Alkalisalze oder Langen aber leicht gefällt werden.

$$
\text { 7-Nitro-7-chlor-6-keto-6,7-dihydroindazol, }
$$

$2 \mathrm{~g}$ Chloroxyindazol löst man in $10 \mathrm{ccm}$ Eisessig und fiigt dann $21 / 2 \mathrm{ccm}$ Salpetersäure $(1,52)$ hinzu, wodurch zunächst Abscheidung des salpetersauren Salzes erfolgt. Man erwärmt nun ganz schwach auf dem Wasserbade, bis gerade alles in Lösung gegangen ist, kühlt dann sofort ab and gibt nach und nach Wasser hinzu. Das Reaktionsprodukt scheidet sich in gelben, derben Kryställchen aus, die abgesaugt und mit verdünnter Essigsäure und schließlich mit Wasser nachgewaschen werden.

Die Verbindung ist unbeständig. Beim Erwärmen 
mit Lösungsmitteln, wie Benzol, Benzin, Eisessig, Alkohol u. dgl. zersetzt sie sich. Auch am Licht erleidet sie leicht Veränderung, indem sie sich braun färbt. Erhitzt man sie für sich, dann fängt sie bei etwa $80^{\circ}$ an sich dunkJer zu färben, und bei $98^{\circ}$ tritt, unter Abgabe von nitrosen Gasen und von Chlor, Zersetzung ein, ohne eigentliches Schmelzen. Das Zersetzungsprodukt ist auch bei $300^{\circ}$ noch nicht geschmolzen.

Zur Analyse wurde die Substanz unter Lichtabschluß bei Zimmertemperatur im Chlorcalciumexsiccator getrocknet.

$0,1359 \mathrm{~g}$ gaben $23,50 \mathrm{ccm}$ Stickgas bei $16^{\circ}$ u. $744 \mathrm{~mm}$ Druck. $0,1515 \mathrm{~g} \quad, \quad 0,0999 \mathrm{AgCl}$.

$\begin{array}{ccc} & \text { Ber. für } \mathrm{C}_{7} \mathrm{H}_{4} \mathrm{O}_{3} \mathrm{~N}_{3} \mathrm{Cl} & \text { Gef. } \\ \mathrm{N} & 19,72 & 19,67 \\ \mathrm{Cl} & 16,60 & 16,30\end{array}$

In Laugen löst sich die Verbindung unter Zersetzung mit tiefgrüner Farbe auf. Säuren scheiden aus der Lösung schmutziggelbe Flocken ab. Die Lösung in konz. Schwefelsäure ist gelb gefärbt. Auf Wasserzusatz erfolgt keine Abscheidung mehr.

Das an und für sich in Benzol schwer lösliche Nitrochlorketon geht beim Erwärmen damit in Lösung. Dabei entweichen nitrose Gase und Chlor und aus der Lösung scheidet sich das im folgenden beschriebene Indazol0 -chinon aus.

Das Nitrochlorketon ist nicht das einzige Produkt der Einwirkung von Salpetersäure auf das Chloroxyindazol. Nebenher entsteht, allerdings nur in sehr geringer Menge, ein Nitrochloroxyindazol, dessen Beschreibung folgt.

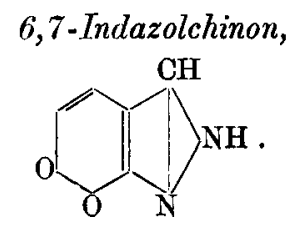

$1 \mathrm{~g}$ des vorher beschriebenen Nitrochlorketodihydroindazols wird mit $60 \mathrm{ccm}$ Benzol karze Zeit auf etwa 
$60^{\circ}$ erwärmt. Von ungelöstem Keton gießt man dann $a b$ und filtriert die Lösung. Das Zurückgebliebene wird alsdann mit $25 \mathrm{ccm}$ frischem Benzol vollends in Lösung gebracht, diese ebenfalls filtriert und die vereinigten Filtrate kurze Zeit zum Sieden erhitzt. Es entweichen nitrose Gase und Chlor, aus der Lösung aber scheidet sich das leuchtend rot gefärbte Indazolchinon ab, das von der noch siedenden Flüssigkeit abfiltriert wird. Man erhält das Chinon so unmittelbar rein. Es ist in Benzol und in Benzin fast unlöslich; in Alkohol löst es sich leicht, in Eisessig, aus dem es sich mit einiger Vorsicht umkrystallisieren läßt, etwas schwerer. Die roten prismatischen Kryställchen fangen bei $160^{\circ}$ an sich dunkelrot zu färben, bei $200^{\circ}$ sehen sie schwarz aus und bei $300^{\circ}$ sind sie noch nicht geschmolzen.

$0,0959 \mathrm{~g}^{1}$ ) gaben $0,1988 \mathrm{CO}_{2}$ und $0,0244 \mathrm{H}_{2} \mathrm{O}$.

$0,0961 \mathrm{~g} \quad 16 \mathrm{ccm}$ Stickgas bei $17^{\circ}$ und $749 \mathrm{~mm}$ Druck. Ber. für $\mathrm{C}_{7} \mathrm{H}_{4} \mathrm{O}_{2} \mathrm{~N}_{2}$

Gef.

$\begin{array}{rrr}\text { C } & 56,71 & 56,54 \\ \text { II } & 2,72 & 2,84 \\ \text { N } & 18,96 & 18,99\end{array}$

I)as hellrote Chinon geht am Licht in eine dunkel braunrote Verbindung über. Scheinbar dieselbe Verbindung entsteht, wenn man das Chinon mit konz. Salzsäure behandelt. Es geht zunächst mit gelber Farbe in Lösung, alsbald scheidet sich aber ein braunrotes Produkt aus, das sich in Alkali mit kräftig blauer Farbe 1öst. Beim Stehen wird diese Lösung olivengrün und schließlich braun. Dieses Verhalten des 6,7-Indazolchinons erinnert an das des Phenylpseudoazimido-1,2-benzochinons, das sich unter ähnlichen Bedingungen auch in ein dunkelrot gefärbtes Produkt verwandelt. Über die Natur dieser schwer löslichen dunkelgefärbten Umwandlungsprodukte können wir zurzeit nichts weiter aussagen, als daß sie noch Chinon-Figenschaften besitzen.

1) Unter Lichtabschluß und bei gewöhnlicher Temperatur im Chlorcalciumexsiccator getrocknet. 
Das Indazolchinon selbst löst sich in verdünnter Natronlauge mit olivengrüner Farbe, die aber bald dunkelgrün und schlieblich braun wird.

Errwärmt man die Lösung des Chinons in Alkohol oder in Eisessig mit Anilin, dann färbt sich die Flüssigkeit dunkelrot, und nach einigem Stehen scheiden sich derbe dunkelrote Krystalle eines Anilidoindazolchinons aus, das aber nicht näher untersucht wurde.

Durch Reduktion geht das Indazolchinon in 6,7-Dioxyindazol über, das mit Salpetersäure das Chinon zurïckliefert.

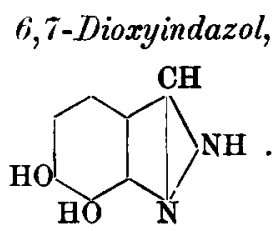

Das in viel Äther verteilte, fein gepulverte Chinon wird mit wäßriger schwefliger Säure versetzt. Bei gelindem Erwärmen und öfterem Schütteln geht alles in Lösung. Man versetzt nun mit Natriumacetat, um die gebildete Schwefelsäure abzustumpfen, hebt den Äther $a b$, schüttelt noch mehrmals mit Äther aus und dunstet dann den Äther zum größten Teil schnell ab. Das Dioxyindazol scheidet sich dabei in derben, farblosen Krysțällchen $a b$, die sich beim Erhitzen langsam violett und später schwarz färben, bei $300^{\circ}$ aber noch nicht geschmolzen sind. In Wasser, Alkohol und in Eisessig leicht löslich, schwerer in Äther, schwer in Benzol und in Benzin.

$0,0999 \mathrm{~g}$ gaben $16,2 \mathrm{ccm}$ Stickgas bei $17^{\circ}$ und $749,5 \mathrm{~mm}$ Druck. Ber. für $\mathrm{C}_{7} \mathrm{H}_{6} \mathrm{U}_{2} \mathrm{~N}_{2}$

N $18,71 \quad 18,51$

An der Luft färbt sich die Dioxyverbindung zunächst schwach violett, später wird sie mibfarbig. Beim Verreiben mit ganz wenig verdünnter Salpetersäure erhält man das Chinon zurück. Die Lösung in Natronlauge färbt sich sofort olivengrün, später blaugrün und dann braun. 
5-Nitro-7-chlor-6-oxyindazol,<smiles>O=[W]1CC2CC3NC(C2Cl)C31</smiles>

Dieses Nitrophenol entsteht in geringer Menge neben dem Nitrochlorketodihydroindazol bei der Einwirkung von Salpetersäure auf Chloroxyindazol. Es findet sich in der Benzolmutterlauge, die bei der Darstellung des Indazolchinons hinterbleibt. Wird dieselbe zum Abdunsten gestellt, dann scheidet sich die Nitroverbindung in kleinen, gelben Kryställchen aus.

Man reinigt sie durch Umkrystallisieren aus Benzol, in dem sie ziemlich schwer löslich ist. Sie schmilzt bei $281^{0}$ unter Zersetzung.

$0,0620 \mathrm{~g}$ gaben $0,0410 \mathrm{AgCl}$.

Ber. für $\mathrm{C}_{7} \mathrm{H}_{4} \mathrm{O}_{3} \mathrm{~N}_{3} \mathrm{Cl} \quad$ Gef.

$\mathrm{Cl}$

16,60

16,35

In Natronlauge löst sich die Verbindung, ohne verändert zu werden, leicht auf. Schon in der Einleitung wurde bemerkt, daß Zweifel bestehen, ob das Nitrochloroxyindazol dem bereits beschriebenen Nitrobromoxyindazol in bezug auf die Stellung der Nitrogruppe und des Halogenatoms wirklich entspricht, wie wir es vorläufig angenommen haben. Denn die Bromverbindung bildet ein schwer lösliches, orangefarbenes Natriumsalz, das sich mit gelber Farbe in Wasser löst. Die Chlorverbindung hingegen löst sich mit gelblichbrauner Farbe in Natronlange und das Natronsalz ist leicht löslich. Eine solche Verschiedenheit ist zum wenigsten sehr auffällig. 